
\title{
3 Research S Quare \\ The 5S rDNA and their chromosomal loci in autotriploid Carassius auratus
}

\section{Liu Cao}

Hunan Normal University

Chun Zhao

Hunan Normal University

Qinbo Qin

Hunan Normal University

Qianhong Gu

Hunan Normal University

Yuwei Zhou

Hunan Normal University

Chongqing Wang

Hunan Normal University

Yude Wang

Hunan Normal University

Huan Qin

Hunan Normal University

Min Tao

Hunan Normal University

Chun Zhang

Hunan Normal University

Rurong Zhao

Hunan Normal University

ShaoJun Liu ( $\square$ Isj@hunnu.edu.cn )

Hunan Normal University https://orcid.org/0000-0001-5761-8571

Research article

Keywords: Autotriploidization; Carassius auratus; Chromosomal locus; Evolution; 5S rDNA

Posted Date: August 31st, 2019

DOI: https://doi.org/10.21203/rs.2.13788/v1 
License: (c) (i) This work is licensed under a Creative Commons Attribution 4.0 International License. Read Full License 


\section{Abstract}

Background Polyploidization can induces a flurry of genetic events and plays an important role in speciation. In this study, we comparatively analyzed the molecular organization and chromosomal locus of $5 S$ rDNA in natural autotriploid $(3 n C C, 3 n=150)$ and artificial autotriploid gynogenetic $(3 n G, 3 n=150)$ Carassius auratus as well as the related diploid ancestors, diploid Carassius auratus $(2 n C C, 2 n=100)$ and Carassius auratus red var. (RCC, $2 n=100$ ), which provide a excellent model to ascertain the genomic changes in autotriploidization process. Results The similarities of the 5S rDNA coding region (class I, class II and class III) between RCC and 3nG were $98.3 \%, 100 \%$ and $99.1 \%$, respectively, and the similarities of the $5 S$ rDNA coding region (class IV, class $V$ and class $\mathrm{VI}$ ) between $2 \mathrm{nCC}$ and $3 \mathrm{nCC}$ were $97.5 \%, 100 \%$ and $100 \%$, respectively. Fluorescence in situ hybridization (FISH) with 5 S rDNA as probe indicated that the expected numbers of maternal chromosomal loci were found in $3 \mathrm{nG}$ and $3 \mathrm{nCC}$, respectively. Conclusions These observations indicate that autotriploidization has little influence on the divergence of 5S rDNA family in Carassius auratus.

\section{Background}

Polyploidy is viewed as an important factor in eukaryote evolution [1-3]. Distant hybridization is a useful tool to produce hybrid offspring with altered genotypes and phenotypes or with different ploidy levels [46]. Fertile triploidy, as a special polyploidy population and a bridge to the formation of tetraploid populations, might play an important role in speciation $[7,8]$. In previous studies, artificial autotriploid gynogenetic offspring ( $3 n=150$, AAA; abbreviated as $3 n G)$ derived from Carassius auratus red var. $(2 \mathrm{n}=100, A \mathrm{~A} ;$ abbreviated as RCC) ( ()$\times$ Megalobrama amblycephala $(2 \mathrm{n}=48, \mathrm{BB}$; abbreviated as BSB) ( () were obtained [9]. The $3 \mathrm{nG}$ are fertile and can produce unreduced eggs (AAA) at an age of 1 year [10]. In the Dongting water system, crucian carp (Carassius auratus L., abbreviated as $\mathrm{CC}$ ) complexes, including diploid $(2 n=100 ; 2 n C C)$ [11], triploid $(3 n=150 ; 3 n C C)$ and tetraploid $(4 n=200 ; 4 n C C)$ forms exist [12]. Triploid $C$. auratus are considered of autotriploid origin [13] and have bisexual fertile and gynogenesis reproduction models [12]. These fertile autotriploids provide an attractive model for the study of the genomic changes.

The $5 \mathrm{~S}$ rDNAs in higher eukaryotes are tandemly arrayed and involve a coding sequence (120bp) and a nontranscribed spacer (NTS) region containing some regulatory elements for coding sequence transcription $[14,15]$. Variation within the NTS region is mainly due to its length and molecular composition [16]. Because of its special structure, the 5S rDNA multigene, is an important maker to analyze and trace rapid evolutionary events. Many 5S rDNA studies have demonstrated the influence of polyploidy on intragenomic variation and gene expression $[17,18]$. The evolutionary dynamics of the $5 S$ rDNA family likely reflect processes of chromosome repatterning that took place during speciation [19, 20]. Therefore, the inheritance pattern and chromosome constitution of 5S rDNAs among polyploid hybrids and their parents can be used to explore the associated hereditary relationships [21]. This study performed a comparative analysis of the 5S rDNA sequence and chromosomal locus in $3 \mathrm{nG}$ and $3 \mathrm{nCC}$ as well as the related diploid ancestors, RCC and 2nCC. 


\section{Methods}

\section{Source of samples}

All experiments were approved by the Animal Care Committee of Hunan Normal University and followed the guidelines statement of the Administration of Affairs Concerning Animal Experimentation of China. Allotetraploid hybrids $\left(4 \mathrm{n}=148\right.$, AABB; abbreviated as $4 \mathrm{nF}_{1}$ ) derived from Carassius auratus red var. $(2 \mathrm{n}=100, \mathrm{AA}$; abbreviated as RCC) ( $(\mathrm{)}) \times$ Megalobrama amblycephala $(2 \mathrm{n}=48, \mathrm{BB}$; abbreviated as $\mathrm{BSB})(\mathbb{\nabla})$ produced autotriploid gametes $(3 n=150, A A A)$ via abnormal chromosome behavior during meiosis [9]. Autotriploid gametes, activated by UV-irradiated BSB sperm, developed into gynogenetic offspring $(3 n=150, A A A ;$ abbreviated as $3 n G)$ [10]. Natural autotriploid Carassius auratus ( $3 n C C, 3 n=150$ ) were collected from the Yuanjiang River and Xiangjiang River in the Dongting water system, Hunan Province. The next generation of $3 \mathrm{nCC}$ were produced by artificial gynogenesis from $3 \mathrm{nCC}$ eggs. Autotriploid Carassius auratus are not endangered or protected species, thus no special permit was necessary for the collection. After blood and the fin tissue were gathered, all samples were kept alive. They were reared and bred at the Reproduction of State Education Ministry, China, located at Hunan Normal University.

\section{PCR amplification and sequencing of the 5S rDNA sequences}

A pair of primers (5SP1, 5'-GCTATGCCCGATCTCGTCTGA-3': 5SP2R,

5'- CAGGTTGGTATGGCCGTAAGC-3') were applied to amplify 5S rDNA products by PCR [18]. Sequences were analyzed with Clustal W [38]. To further evaluate differences in 5S rDNA patterns, 210 clones were sequenced, containing 30 clones from RCC (10 clones for each PCR fragment), 60 clones from 3nG (20 clones for each PCR fragment), 60 clones from the $2 \mathrm{nCC}$ (20 clones for each PCR fragment) and 60 clones from the $3 \mathrm{nCC}$ ( 20 clones for each PCR fragment).

\section{Preparation of chromosome spreads}

Chromosome preparation was carried out on the fin cell of RCC, $3 n G, 2 n C C$ and $3 n C C$. The fin cell culture and chromosome preparation was performed according to the method reported by Zhu et al. [39] and Earley [40] with minor modifications. Three hours prior to harvest the cells was added concanavalin $(0.1 \mu \mathrm{g} / \mathrm{mL})$. The collected cells were ground in $0.0375 \% \mathrm{NaCl}$, followed by hypotonic treatment with $0.075 \mathrm{M} \mathrm{KCl}$ at $28^{\circ} \mathrm{C}$ for $15-20 \mathrm{~min}$ and then fixed in 3:1 methanol-acetic acid for 20-30 min with two changes. Chromosome preparations were examined under an oil lens at a magnification of 3330x. For each fish sample, 200 metaphase spreads ( 20 metaphase spreads in RCC, $3 n G, 2 n C C$ and $3 n C C$ ) were examined.

\section{Fluorescence in situ Hybridization}

The $5 \mathrm{~S}$ gene probes for fluorescence in situ hybridization (FISH) were amplified from RCC (203bp, 340bp and 477bp) and 2nCC (203bp, 340bp and 490bp) by PCR using 5SP1 and 5SP2R primers labeled with Dig-11-dUTP (using a nick translation kit; Roche, Germany). FISH was performed according to the method 
described by Qin et al. [41]. For each fish sample, 200 metaphase spreads (20 metaphase spreads in each sample) of the chromosomes were analyzed. Preparations were examined under an inverted microscope (CW4000, Leica, Germany) with a confocal imaging system (LCS SP2, Leica). Captured images were colored and superimposed in Adobe Photoshop CS6.

\section{Results}

\section{Examination of chromosome number}

In RCC individuals, $87.5 \%$ of the chromosomal metaphases had 100 chromosomes (Figure. 1a). In 3nG individuals, $90 \%$ of the chromosomal metaphases had 150 chromosomes (Figure. 1b). In 2nCC individuals, $89.5 \%$ of the chromosomal metaphases had 100 chromosomes (Figure. 1c). In 3nCC individuals, $94.5 \%$ of the chromosomal metaphases had 150 chromosomes (Figure. 1d).

\section{S rDNA sequences}

Three 5S PCR fragments (approximately 200, 340, and $500 \mathrm{bp}$ ) were amplified from the genomes of RCC, $3 n G, 2 n C C$ and $3 n C C$ (Figure. 2). Based on the BLASTn analysis, all fragments from RCC, 3nG, 2nCC and 3nCC were confirmed as 5 S rDNA repeat units, comprising a complete 5 S rDNA region and a whole NTS region (Figure. 3). In RCC, three monomeric 5S rDNA (designated class I, class II and class III) were characterized by distinct NTS types (designated NTS-I: 83-bp, NTS-Il: 220-bp and NTS-III: 357-bp). 3nG had three monomeric 5S rDNAs inherited from RCC (Figure. 4a and Figure. 5a-c). The coding region similarities (class I, class II and class III) between RCC and 3nG were $98.3 \%, 100 \%$ and $99.1 \%$, respectively. In 2nCC, three monomeric $5 \mathrm{~S}$ rDNAs (designated class IV, class $\mathrm{V}$ and class $\mathrm{VI}$ ) possessed three NTS types (designated NTS-IV: 83-bp, NTS-V: 220-bp and NTS-VI: 370-bp). 3nCC had three monomeric 5S rDNAs, which were completely from 2nCC (Figure. 4b) and Figure. 5d-f). The coding region similarities (class IV, class V and class VI) between 2nCC and 3nCC were $97.5 \%, 100 \%$ and $100 \%$, respectively. The accession numbers of $5 S$ rDNA sequences in GenBank are listed in Table 1.

\section{Fluorescence in situ Hybridization}

The 5S rDNA probe (class I: $203 \mathrm{bp}$ ) hybridized with the metaphase chromosomes of RCC and 3nG. The $5 S$ rDNA probe (class IV: $203 \mathrm{bp}$ ) hybridized with the metaphase chromosomes of $2 \mathrm{nCC}$ and $3 \mathrm{nCC}$. The FISH results showed that eight 5S rDNA gene loci were present in 2nCC and RCC (Figure. 6a, c, respectively; Table 2). Twelve 5S rDNA gene loci were present in $3 \mathrm{nG}$ and $3 \mathrm{nCC}$ (Figure. 6b, d, respectively; Table 2).

The 5S rDNA probe (class II: $340 \mathrm{bp}$ ) hybridized with the metaphase chromosomes of RCC and $3 \mathrm{nG}$. The $5 S$ rDNA probe (class V: $340 \mathrm{bp}$ ) hybridized with the metaphase chromosomes of $2 \mathrm{nCC}$ and $3 \mathrm{nCC}$. The FISH results showed that four $5 \mathrm{~S}$ rDNA loci were present in 2nCC and RCC (Figure. 7a, c, respectively; Table 2). Six 5S rDNA loci were found in 3nG and 3nCC (Figure. 7b, d, respectively; Table 2). 
The 5S rDNA probe (class III: $477 \mathrm{bp}$ ) hybridized with the metaphase chromosomes of RCC and 3nG. The $5 \mathrm{~S}$ rDNA probe (class Vl: $490 \mathrm{bp}$ ) hybridized with the metaphase chromosomes of $2 \mathrm{nCC}$ and $3 \mathrm{nCC}$. The FISH results showed that eight $5 \mathrm{~S}$ rDNA gene loci were identified in 2nCC and RCC (Figure. 8a, c, respectively; Table 2). Twelve 5S rDNA gene loci were identified in $3 \mathrm{nG}$ and $3 \mathrm{nCC}$ (Figure. 8b, $d$, respectively; Table 2).

\section{Discussion}

Birth-and-death and concerted evolution are important mechanisms in driving the long-term evolution of $5 S$ rDNA [22]. Generally, fish genome has two $5 S$ rDNA arrays $[23,24]$. However, three $5 S$ rDNA arrays were identified in RCC and 2nCC. In teleosts, fish-specific genome duplication has been revealed [25-27]. All $C$. auratus (Cypriniformes, Cyprinidae) karyotypes and genome sizes are approximately twice as large as those of standard diploid cyprinid fish [11,28-30], which are viewed as undergoing an initial polyploidization process [31]. Reorganizations and variations of genomes in the polyploidization process probably lead to new 5 S rDNA-arrays [18].

In this paper, three $5 S$ rDNA classes of $3 n G$ and $3 n C C$ were completely inherited from RCC and 2nCC, respectively. The mapping of tandemly repeated sequences by means of FISH has proven to be very useful in analyzing fish genome architectures and large amounts of evolutionary, ecological and taxonomic questions [32]. The results of FISH showed that the 5S rDNA clusters (class I, class II and class III) were localized in the chromosomes of $3 \mathrm{nG}$ and the $5 \mathrm{~S}$ rDNA clusters (class IV, class V and class $\mathrm{VI}$ ) were localized in the chromosomes of $3 \mathrm{nCC}$. Recurrent autopolyploidization has been reported to occur in the $C$. auratus complex [33]. The extant triploid form of the $C$. auratus complex with more than 150 chromosomes has undergone more than one genome polyploidy event and occurred on the basis of previous polyploidization [34]. Interestingly, it is difficult to distinguish $2 \mathrm{nCC}$ and $3 \mathrm{nCC}$ by appearance, and their mitochondrial DNA show high similarity [12]. Therefore, it is reasonable to infer that autotriploidization has little influence on the divergence of the $5 S$ rDNA family in $C$. auratus.

Considering the general behavior of other polyploids, some changes following polyploidy were extremely probably to have occurred. Previous information has indicated that the uniparental loss of 5S rDNA classes and the variation of $5 \mathrm{~S}$ rDNA chromosomal loci from progenitors have been detected in polyploid progeny $[19,35,36]$. These variations possibly reflect instability in polyploid genomes. However, maternal genome is stably inherited in progeny by the method of gynogenesis in autotriploid $C$. auratus. The conservation of $5 \mathrm{~S}$ rDNA molecular organization and chromosomal loci was detected in $3 \mathrm{nG}$ and $3 \mathrm{nCC}$. The high conservation in rDNA site number and chromosomal location, likely indicates conserved genome organization [37]. Thus, we speculate that the unique reproduction mode may allows them to reduce the pressure of evolution, resulting in the genetic conservation.

Because of the genetic conservation of $5 \mathrm{~S}$ rDNA in 3nG and 3nCC, 5S rDNA is a useful genomic DNA maker. In our study, 3nG was derived from the hybridization of RCC $(\nabla) \times B S B(\nabla)$ which had a clear formation pathway. The $3 \mathrm{nCC}$ was derived from the crossing of diploid $C$. auratus $(\mathbb{\nabla})$ with different 
species $(\llbracket)[12,13]$. However, it is still lack of clear molecular evidence to support this. The similar genetic characteristics of the $5 \mathrm{~S} \mathrm{rDNA}$ in $3 \mathrm{nCC}$ and $3 \mathrm{nG}$ revealed that $3 \mathrm{nCC}$ was of hybrid origin with the similar diploid ancestors as occurred in $3 \mathrm{nG}$, which endorsed the viewpoint of the hybrid origin's natural autotriploid $C$. auratus.

\section{Conclusions}

The molecular organization and chromosomal locus of $5 \mathrm{~S}$ rDNA showed conservation in $3 \mathrm{nCC}$ and $3 \mathrm{nG}$. These data indicate that autotriploidization has little influence on the divergence of 5S rDNA family in $C$. auratus, which extend the knowledge of evolutionary dynamics of the 5S rDNA family in autotriploid fishes and have great significance in the study of Carassius taxa evolution.

\section{Abbreviations}

3nCC: Natural autotriploid Carassius auratus; 3nG: Artificial autotriploid gynogenetic offspring; 2nCC: Diploid Carassius auratus; RCC: Carassius auratus red var.; BSB: Megalobrama amblycephala; $4 \mathrm{nF}_{1}$ : Allotetraploid hybrids; NTS: Nontranscribed spacer; FISH: Fluorescence in situ Hybridization.

\section{Declarations}

\section{Ethics approval and consent to participate}

All experiments were approved by the Animal Care Committee of Hunan Normal University and followed the guidelines statement of the Administration of Affairs Concerning Animal Experimentation of China.

\section{Consent to publish}

Not applicable.

\section{Authors' contributions}

S.J.L. contributed to the conception and designed the study; L.C, C.Z., Q.B.Q. carried out the experimental work, participated in rafted the manuscript. Q.H.G., Y.W.Z., C.Q.W., Y.D.W., H.Q. analyzed sequences and M.T., C.Z., R.R.Z. participated in interpretation and discussion of the results. All authors read and approved the final manuscript.

\section{Availability of data and materials}

The complete clean reads for these libraries have been uploaded to the NCBI Sequence Read Archive site (http://www.ncbi.nlm.nih.gov/sra/; accession nos. GQ485555, GQ485556, GQ485557, MK073481, MK073482, MK073483, MK073487, MK073488, MK073489, MK073484, MK073485, MK073486.

\section{Competing interests}


The authors declare that they have no competing interests.

\section{Funding}

This work was supported by the National Natural Science Foundation of China(Grant Nos. 31430088 and 31730098), the earmarked fund for China Agriculture Research System (Grant No. CARS-45), the Hunan Provincial Natural Science and Technology Major Project (GrantNo. 2017NK1031), the Key Research and Development Program of Hunan Province (Grants No. 2018NK2072), the Natural Science Foundation of Hunan Province for DistinguishedYoung Scholars (Grant No. 2017JJ1022), the Major Program of the EducationalCommission of Hunan Province (Grant No. 17A133), and the Cooperative Innovation Center of Engineering and New Products for Developmental Biologyof Hunan Province (Grant No. 20134486). The funding body played no role in the design of the study, collection, analysis, and interpretation of data and in writing the manuscript.

\section{Acknowledgments}

We would like to sincerely thank many researchers who help to complete this manuscript.

\section{References}

1. Comai L: Genetic and epigenetic interactions in allopolyploid plants. Plant Molecular Biology 2000, 43(2-3):387-399.

2. Liu S, Liu Y, Zhou G, Zhang X, Luo C, Feng H, He X, Zhu G, Yang H: The formation of tetraploid stocks of red crucian carp $X$ common carp hybrids as an effect of interspecific hybridization. Aquaculture 2001, 192(2):171-186.

3. Peer YVD, Maere S, Meyer A: The evolutionary significance of ancient genome duplications. Nature Reviews Genetics 2009, 10(10):725-732.

4. Bullini L: Origin and evolution of animal hybrid species. Trends in Ecology \& Evolution 1994, 9(11):422-426.

5. Liu Q, Qi Y, Liang Q, Xu X, Hu F, Wang J, Xiao J, Wang S, Li W, Tao M: The chimeric genes in the hybrid lineage of Carassius auratus cuvieri $(\mathbb{\nabla}) \times$ Carassius auratus red var. ( $(\mathbb{Z})$. Science China Life Sciences 2018, 61(9):1-11.

6. Mallet J: Hybrid speciation. Nature 2007, 446(7133):279-283.

7. Husband BC: The role of triploid hybrids in the evolutionary dynamics of mixed-ploidy populations. Biological Journal of the Linnean Society 2004, 82(4):537-546.

8. Kovalsky IE, Roggero Luque JM, Elías G, Fernández SA, Solís Neffa VG: The role of triploids in the origin and evolution of polyploids of Turnera sidoides complex (Passifloraceae, Turneroideae[J]. Journal of Plant Research 2018. 
9. Qin Q, Wang Y, Wang J, Dai J, Liu Y, Liu S: Abnormal chromosome behavior during meiosis in the allotetraploid of Carassius auratus red var. (邓) $\times$ Megalobrama amblycephala (邓). Bmc Genetics 2014, 15(1):95-95.

10. Qin Q, Wang J, Dai J, Wang YD, Liu Y, Liu S: Induced All-Female Autotriploidy in the Allotetraploids of Carassius auratus red var. ( $(\mathbb{\nabla}) \times$ Megalobrama amblycephala $(\mathbb{\nabla})$. Marine Biotechnology 2015, 17(5):604-612.

11. Zan R: Analysis and comparison between the karyotypes of Cyprinus carpio and Carassius auratus as well as Aristichthys nobilis and Hypophthalmichthys molitrix. Acta Genetica Sinica 1980, 7(1):7277.

12. Xiao J, Zou T, Chen Y, Chen L, Liu S, Tao M, Zhang C, Zhao R, Zhou Y, Long Y: Coexistence of diploid, triploid and tetraploid crucian carp (Carassius auratus) in natural waters. Bmc Genetics 2011, 12(1):20.

13. Qin Q, Wang J, Hu M, Huang S, Liu S: Autotriploid origin of Carassius auratus as revealed by chromosomal locus analysis. Science China Life Sciences 2016, 59:622-626.

14. Nielsen JN, Hallenberg C, Frederiksen S, Sørensen PD, Lomholt B, . Transcription of human 5S rRNA genes is influenced by an upstream DNA sequence. Nucleic Acids Research 1993, 21(16):3631-3636.

15. Pasolini P, Costagliola D, Rocco L, Tinti F: Molecular organization of 5S rDNAs in Rajidae (Chondrichthyes): Structural features and evolution of piscine 5S rRNA genes and nontranscribed intergenic spacers. Journal of Molecular Evolution 2006, 62(5):564-574.

16. Barman AS, Singh M, Singh RK, Lal KK: Evidence of birth-and-death evolution of $5 S$ rRNA gene in Channa species (Teleostei, Perciformes). Genetica 2016, 144(6):723-732.

17. Adams KL, Wendel JF: Polyploidy and genome evolution in plants. Current Opinion in Plant Biology 2005, 8(2):135-141.

18. Qin Q, He W, Liu S, Wang J, Xiao J, Liu Y: Analysis of 5S rDNA organization and variation in polyploid hybrids from crosses of different fish subfamilies. Journal of Experimental Zoology Part B Molecular \& Developmental Evolution 2010, 314(5):403-411.

19. Qin Q, Lai Z, Cao L, Xiao Q, Wang Y, Liu S: Rapid genomic changes in allopolyploids of Carassius auratus red var.(殴 $\times$ Megalobrama amblycephala(囚). Scientific Reports 2016, 6(1):34417.

20. Sember A, Bohlen J, Šlechtová V, Altmanová M, Pelikánová Š, Ráb R: Dynamics of tandemly repeated DNA sequences during evolution of diploid and tetraploid botiid loaches (Teleostei: Cobitoidea: Botiidae). Plos One 2018, 13(3):e0195054.

21. Qin Q, Huo Y, Liu Q, Wang C, Zhou Y, Liu S: Induced gynogenesis in autotetraploids derived from

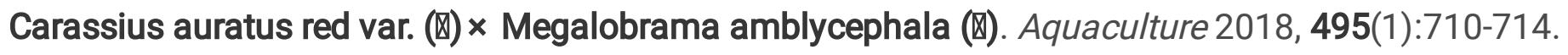

22. Campo D, Machado-Schiaffino G, Horreo JL, Garcia-Vazquez E: Molecular organization and evolution of $5 \mathrm{~S}$ rDNA in the genus Merluccius and their phylogenetic implications. Journal of Molecular Evolution 2009, 68(3):208-216.

23. Martins C, Galetti Jr PM: Two 5S rDNA arrays in Neotropical fish species: is it a general rule for fishes? Genetica 2001, 111:439-446. 
24. Martins C, Wasko AP, Oliveira C, Wright JM: Nucleotide Sequence of 5s rDNA and Localization of the Ribosomal RNA Genes to Metaphase Chromosomes of the Tilapiine Cichlid Fish, Oreochromis Niloticus. Hereditas 2004, 133(1):39-46.

25. Jaillon O, Aury J, Brunet F, Petit J, Stange-Thomann N, Mauceli E, Bouneau L, et al. Genome duplication in the teleost fish Tetraodon nigroviridis reveals the early vertebrate proto-karyotype. Nature 2004, 431(7011):946-957.

26. Venkatesh B: Evolution and diversity of fish genomes. Current Opinion in Genetics \& Development 2003, 13(6):588-592.

27. Taylor JS, Braasch I, Frickey T, Meyer A, Peer YVD: Genome Duplication, a Trait Shared by 22,000 Species of Ray-Finned Fish. Genome Research 2003, 13(3):382-390.

28. Ohno S: Evolution by Gene Duplication: Springer Berlin Heidelberg; 1970.

29. Ohno S, Atkin NB: Comparative DNA values and chromosome complements of eight species of fishes. Chromosoma 1966, 18(3):455-466.

30. Risinger C, Larhammar D: Multiple loci for synapse protein SNAP-25 in the tetraploid goldfish. Proceedings of the National Academy of Sciences of the United States of America 1993, 90(22):10598-10602.

31. Luo J, Gao Y, Ma W, Bi X, Wang S, Wang J, et al. Tempo and mode of recurrent polyploidization in the Carassius auratus species complex (Cypriniformes, Cyprinidae). Heredity 2014, 112(4):415-427.

32. Symonová R, Howell WM: Vertebrate Genome Evolution in the Light of Fish Cytogenomics and rDNAomics. Genes 2018, 9(2):96.

33. Gao Y, Wang S-Y, Luo J, Murphy RW, Du R, Wu S-F, et al. Quaternary palaeoenvironmental oscillations drove the evolution of the Eurasian Carassius auratus complex (Cypriniformes, Cyprinidae). Journal of Biogeography 2012, 39(12):2264-2278.

34. Jiang FF, Wang ZW, Zhou L, Jiang L, Zhang XJ, Apalikova OV, et al. High male incidence and evolutionary implications of triploid form in northeast Asia Carassius auratus complex. Molecular Phylogenetics \& Evolution 2013, 66(1):350-359.

35. He W, Qin Q, Liu S, Li T, Jing W, Xiao J, et al. Organization and Variation Analysis of 5S rDNA in Different Ploidy-level Hybrids of Red Crucian Carp × Topmouth Culter. Plos One 2012, 7(6):e38976.

36. Hu F, Wu C, Zhou Y, Cao L, Xiao J, Wang S, et al. Production of androgenetic, triploid and tetraploid hybrids from the interspecific hybridization of female Japanese crucian carp and male blunt snout bream. Aquaculture 2018, 491.

37. Bo L, Thomas MD: Conservation and Loss of Ribosomal RNA Gene Sites in Diploid and Polyploid Fragaria (Rosaceae). BMC Plant Biology, 2011, 11:157.

38. Thompson JD, Higgins DG, Gibson TJ: CLUSTAL W: improving the sensitivity of progressive multiple sequence alignment through sequence weighting, position-specific gap penalties and weight matrix choice. In: Nucleic Acids Res: 1994. 4673-4680. 
39. Zhu DM, Yang K, Wang WM, Song W: Establishment and characterization of a fin cell line from blunt snout bream, Megalobrama amblycephala. Fish Physiology \& Biochemistry 2013, 39:1399-1410.

40. Earley EM: Chromosome preparations from monolayer cell cultures. Methods Cell Sci 1975, 1:31-35.

41. Qin Q, Liu Q, Wang C, Cao L, Zhou Y, Qin H, et al. Molecular Organization and Chromosomal Localization Analysis of $5 \mathrm{~S}$ rDNA Clusters in Autotetraploids Derived From Carassius auratus Red

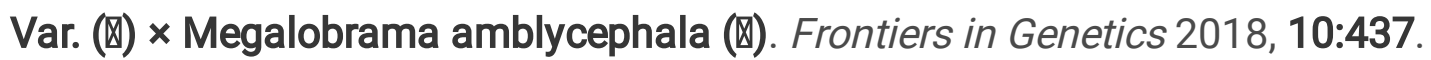

\section{Tables}

Table 1 GenBank accession numbers of the 5S rDNA sequences in RCC, 3nG, $2 \mathrm{nCC}$ and $3 \mathrm{nCC}$

\begin{tabular}{|c|c|c|c|c|c|c|}
\hline \multirow[b]{2}{*}{ Fish type $^{\mathrm{a}}$} & \multicolumn{4}{|c|}{ DNA fragments (bp) } & \multirow[b]{2}{*}{492} & \multirow[b]{2}{*}{495} \\
\hline & 203 & 340 & 477 & 490 & & \\
\hline$\overline{\mathrm{RCC}}$ & GQ485555 & GQ485556 & GQ485557 & \multirow{4}{*}{ MK073489 } & & \multirow{4}{*}{ MK073483 } \\
\hline $3 n G$ & MK073481 & MK073482 & & & & \\
\hline $2 \mathrm{nCC}$ & MK073487 & MK073488 & & & & \\
\hline 3nCC & MK073484 & MK073485 & & & MK073486 & \\
\hline
\end{tabular}

aRCC, Carassius auratus red var.; 3nG, Autotriploid gynogenetic offspring; 2nCC, Diploid Carassius auratus; 3nCC, Autotriploid Carassius auratus

Table 2 Examination of hybridising signals by FISH in RCC, 3nG, 2nCC and 3nCC

\begin{tabular}{ccccccc}
\hline Fish type $^{\mathrm{a}}$ & No. of Fish & No. of metaphases & $203 \mathrm{bp}$ & $340 \mathrm{bp}$ & $477 \mathrm{bp}$ & $490 \mathrm{bp}$ \\
\cline { 4 - 6 } & & & No. of loci & No. of loci & No. of loci & No. of loci \\
\hline RCC & 10 & 200 & 8 & 4 & 8 & \\
\hline 3nG & 10 & 200 & 12 & 6 & 12 & \\
\hline 2nCC & 10 & 200 & 8 & 4 & & 8 \\
\hline 3nCC & 10 & 200 & 12 & 6 & & 12 \\
\hline
\end{tabular}

aRCC, Carassius auratus red var.; 3nG, Autotriploid gynogenetic offspring; 2nCC, Diploid Carassius auratus; 3nCC, Autotriploid Carassius auratus

\section{Figures}



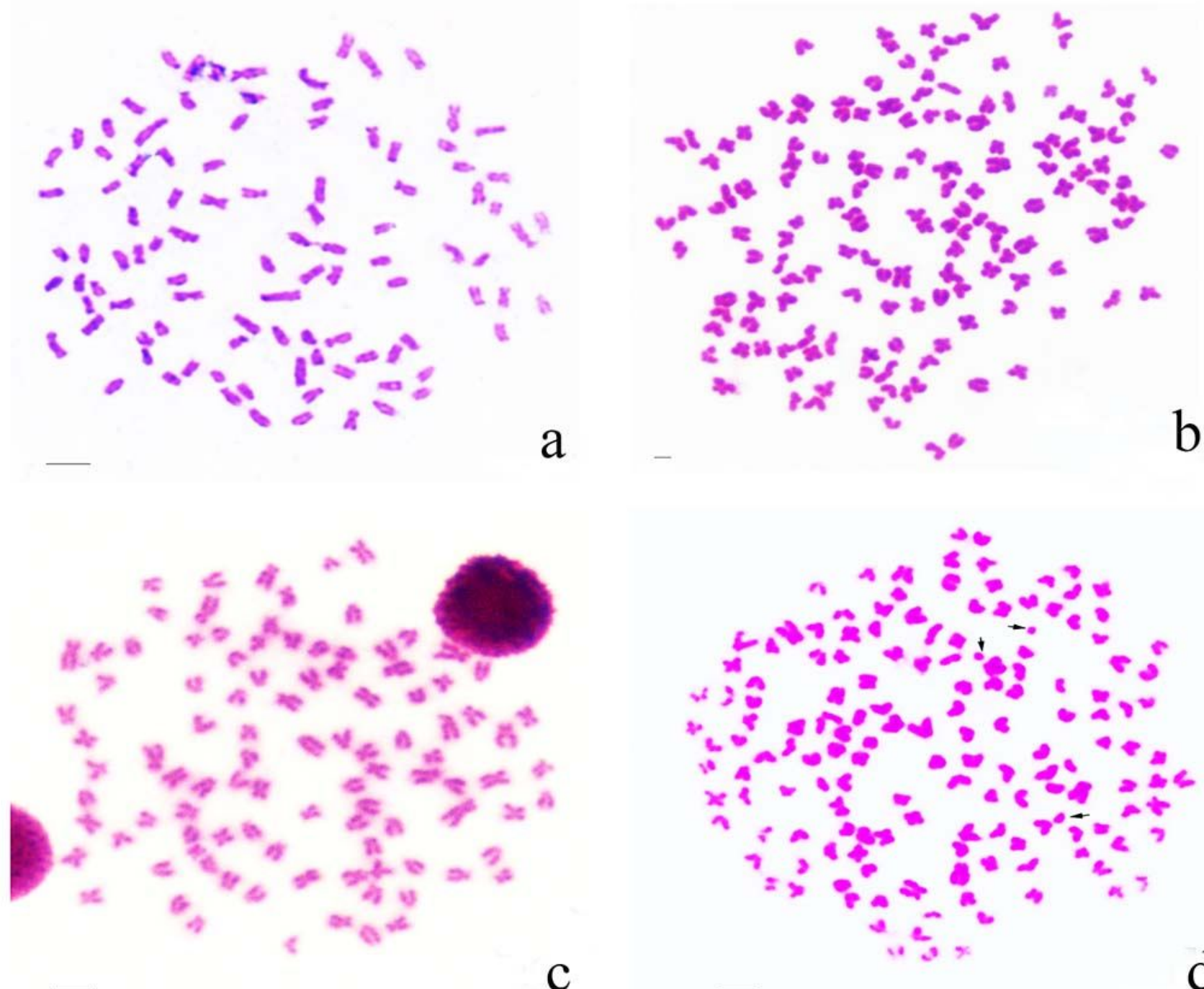

$\mathrm{C}$

d

Figure 1

Chromosome spreads at metaphase in RCC, 3nG, 2nCC and 3nCC (a) The metaphase chromosome spreads of RCC individuals possess 100 chromosomes; (b) The metaphase chromosome spreads of $3 \mathrm{nG}$ individuals possess 150 chromosomes; (c) The metaphase chromosome spreads of $2 \mathrm{nCC}$ individuals possess 100 chromosomes; (d) The metaphase chromosome spreads of 3nCC individuals possess 150 chromosomes, in some samples uncounted micro-chromosomes were found (indicated by arrows). 


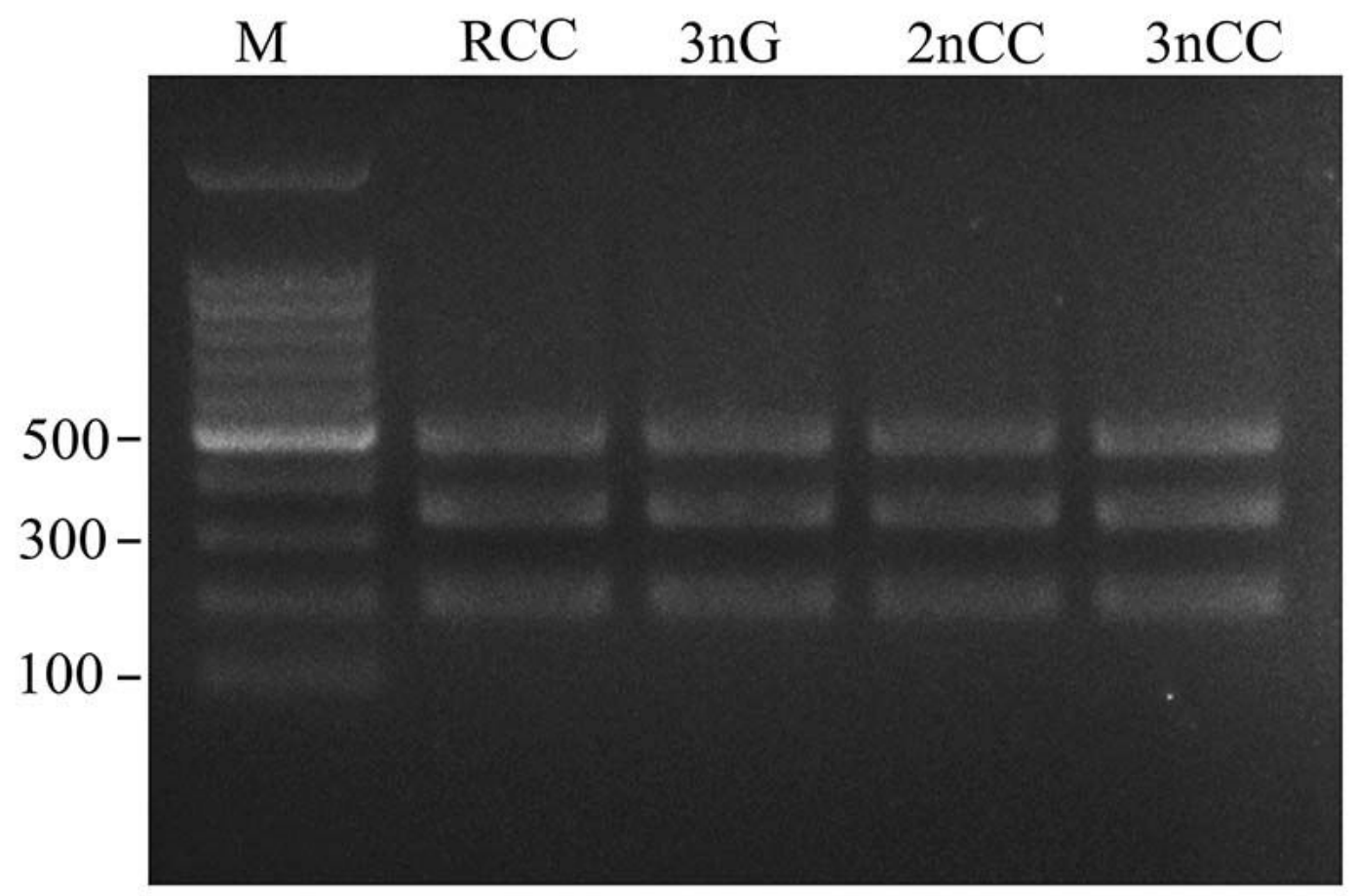

Figure 2

Using the primer pair 5SP1-5SP2R, DNA fragments were amplified from RCC, 3nG, 2nCC and 3nCC. M, DNA ladder markers (100 bp increments); There were three DNA fragments (approximately 200, 340, and 500 bp) from RCC (lane 1), 3nG (lane 2), 2nCC (lane 3) and 3nCC (lane 4). 
(Class I) RCC GCTTACGGCC ATACCAACCT GGCTATGCCC GATCTCCTCT GATCTCGGAA GCTAAGCAGG TTTGGGCCTG GTTAGTACTT (Class II) RCC GCTTACGGCC ATACCAACCT GGCTATGCCC GATCTCOTCT GATCTCGGAA GCTAAGCAGG TTTGGGCCTG GTTAGTACT (Class I) 3ng GCTTACGGCC ATACCAACCT GGCTATGCCC GATCTCGTCT GATCTCGGAA GCTAAGCAGG TTTGGGCCTG GTTAGTACTT (Class II) 3nG GCTTACGGCC ATACCAACCT GGCTATGCCC GATCTCGTCT GATCTCGGAA GCTAAGCAGG TTTGGGCCTG GTTAGTACTATT

(C1ass I) RCC (Class II) RCC

(Class III) RCC

(Class II) 3nG

(C1ass III) 3nG

(C1ass I) RCC (Class II) RCC (Class III) RCC (Class II) 3 no (Class III) 3no

(C1ass I) RCC (C1ass II) RCC (C1ass I) 3n (C1ass II) 3nG (Class III) 3nG

(Class I) RCC (Class II) RCC (Class III) RCC (Class I) 3nG (Class III) 3nG

(Class I) RCC (C1ass III) RCC (Class I) 3nG (Class II) $3 \mathrm{n}$

(C1ass I) RCC (Class II) RCC (Class III) RCC TTTGGAAATT TTTCACTTAG TATATAATAA тTTTGCCAA GATGGGAGA CCGCCTGGGA ATACCAGGTG CTGTAAGCT -19-0. TGGGAGA CCOCCTGGGA ATACCGGOTG СTGTAAGCTT TTTGGACATT TTTCACTTAG TATATAATAA TTTTCCCAA CCAGGTGCTG TAAGCTTTTT GGACATTTTT CACTTAGTAT ATAATAATTT TGCC:CCACTTAGTAT ATAATAATTT TGCC-1 CCAGGTGCTT TAATCTTTTT GGAAAATTT- CACGAATTAT ATAATAATCT TTCATTAAAA AAAAAAAAAA AAAAAAAAAA

AGAGTCAATG CCCGATCTCT GAATCTTAGC AGGTTTAGGT CTGGTTAGAA CTTTGATGAG AGACTGCCTG GGAATACCAG AGAGTCAATG CCCGATCTCT GAATCTTAGC AGGTTTAGGT CTGGTTAGTA CTTTGATGAG AGACTGCCTA GGAATACCAG AOAOTCAATG CCCOATCTCT GAaTctitage

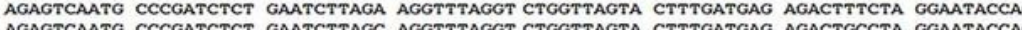

--TTGGGGT TTCTTTCCTA CTTATATAAT GTACTGGCGA TTAGATTGGC TGGTCTTTAA ATAGCCCTC TGCTTTAAG CTTTTGGCTT TTCTTTCCTA CTTATATAAT GTACTGGCGA TAAGATTGGC TGGTCTTTAA ATAGCCCTC GTCCTTTAAG CTTTTGGGTT TTCTTTTCTA CTTATATAAT GTACTGGCGA TTAGATTGGC TGGTCTTTAA ATAGCCCTC TTCGTCAAAA TTGATCTAAT ATACTGCAGA TTAGGGTGGC TGATCTTTAA ATAGCCCACA GTGCTTTAAG CTTTTGGGCT TTCTTTCCTA CTTATATAAT GTACTGGCGA TAAGATTGGC TGCTCTTTAA ATAGCCCTCT CTTTTAG CTTTTGGGTT TTCTTTCCTA CTTATATAAT GTACTATCGA TTAGATTGGC TGGTCTTTAA ATAGCCCTC

СтTTGCAGCA GTCTTC

CTTTGCAGCA GACTTC

CTTTGCAGCT GTCTTC

CTTTGCAGCA GCTCTC

(Class III) 3 G CTTTGCACCA GTCTTC

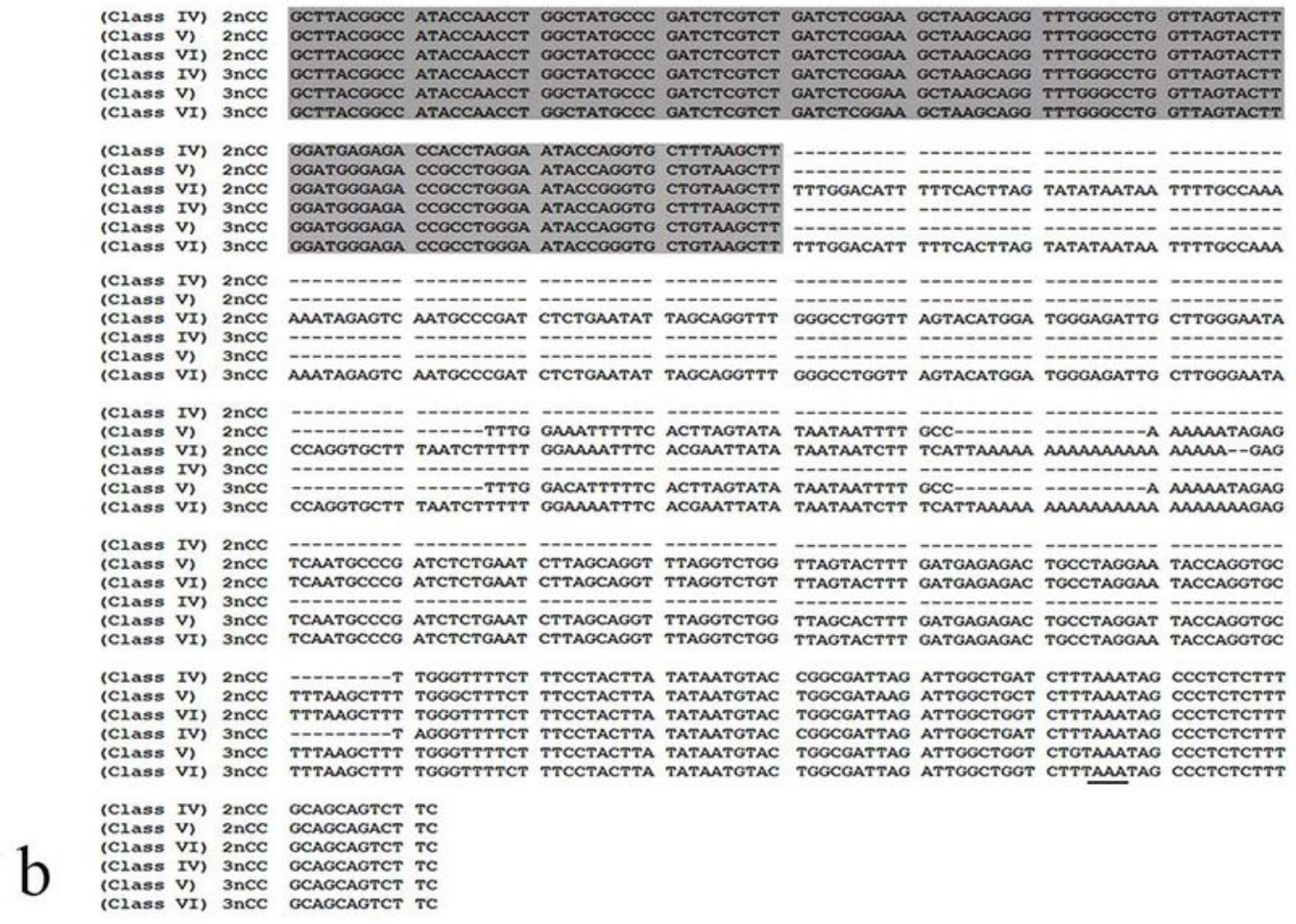

Figure 3

Representative sequences of $5 \mathrm{~S}$ rDNA-classes from RCC, 3nG, 2nCC and 3nCC (a) Representative sequences of 5 S rDNA class I, II, and III from RCC and 3nG; (b) Representative sequences of 5S rDNA class IV, V, and VI from $2 \mathrm{nCC}$ and $3 \mathrm{nCC}$. Complete $5 \mathrm{~S}$ coding regions are shaded; the NTS upstream TATA elements are underlined. 


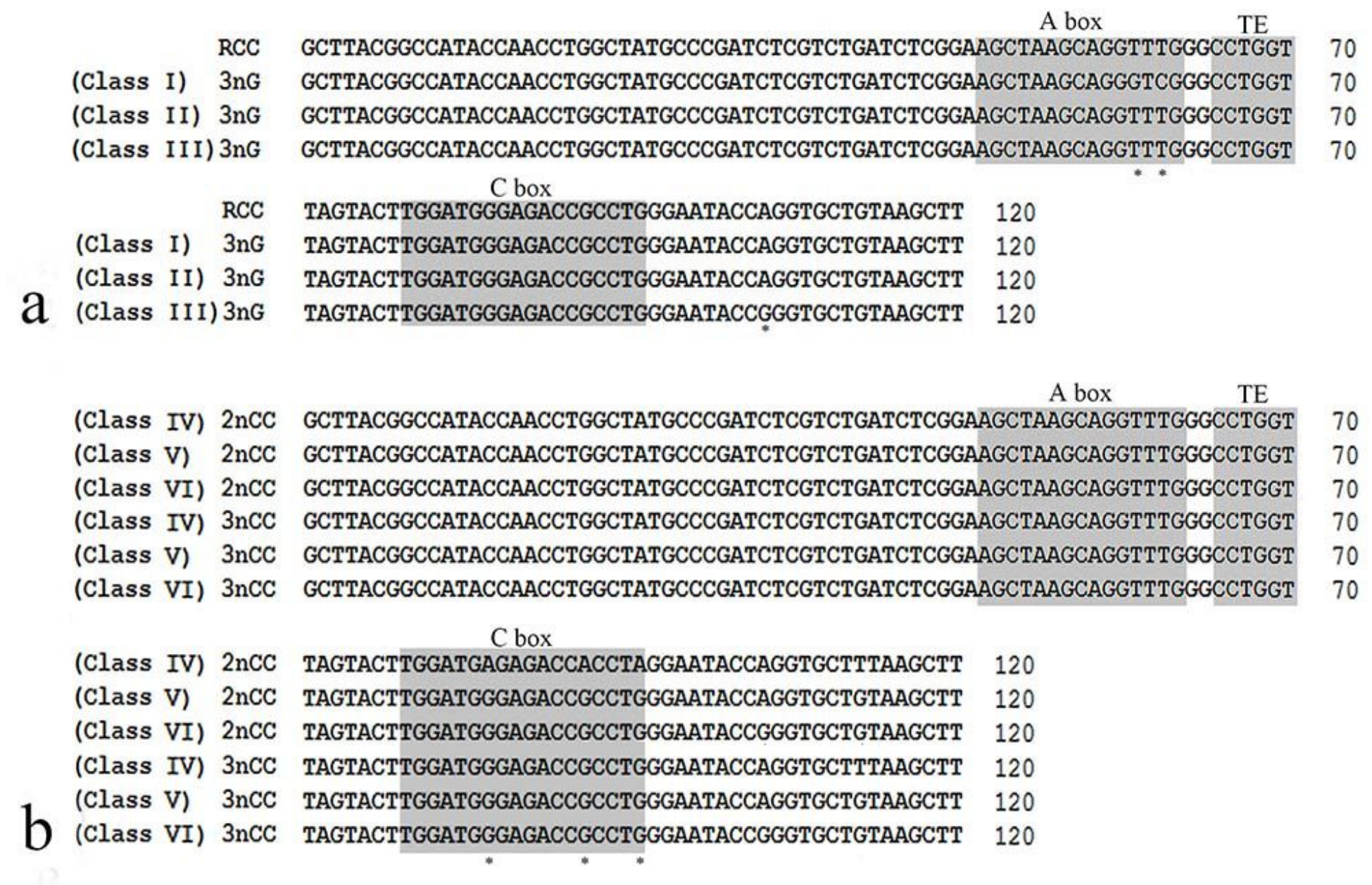

Figure 4

Comparison of $5 \mathrm{~S}$ coding regions from RCC, 3nG, 2nCC and 3nCC (a) 5S coding regions from RCC and $3 n G$; (b) 5 S coding regions from $2 \mathrm{nCC}$ and $3 \mathrm{nCC}$. Asterisks mark variable sites of the $5 \mathrm{~S}$ coding regions. Internal control regions of the coding region are shaded. 


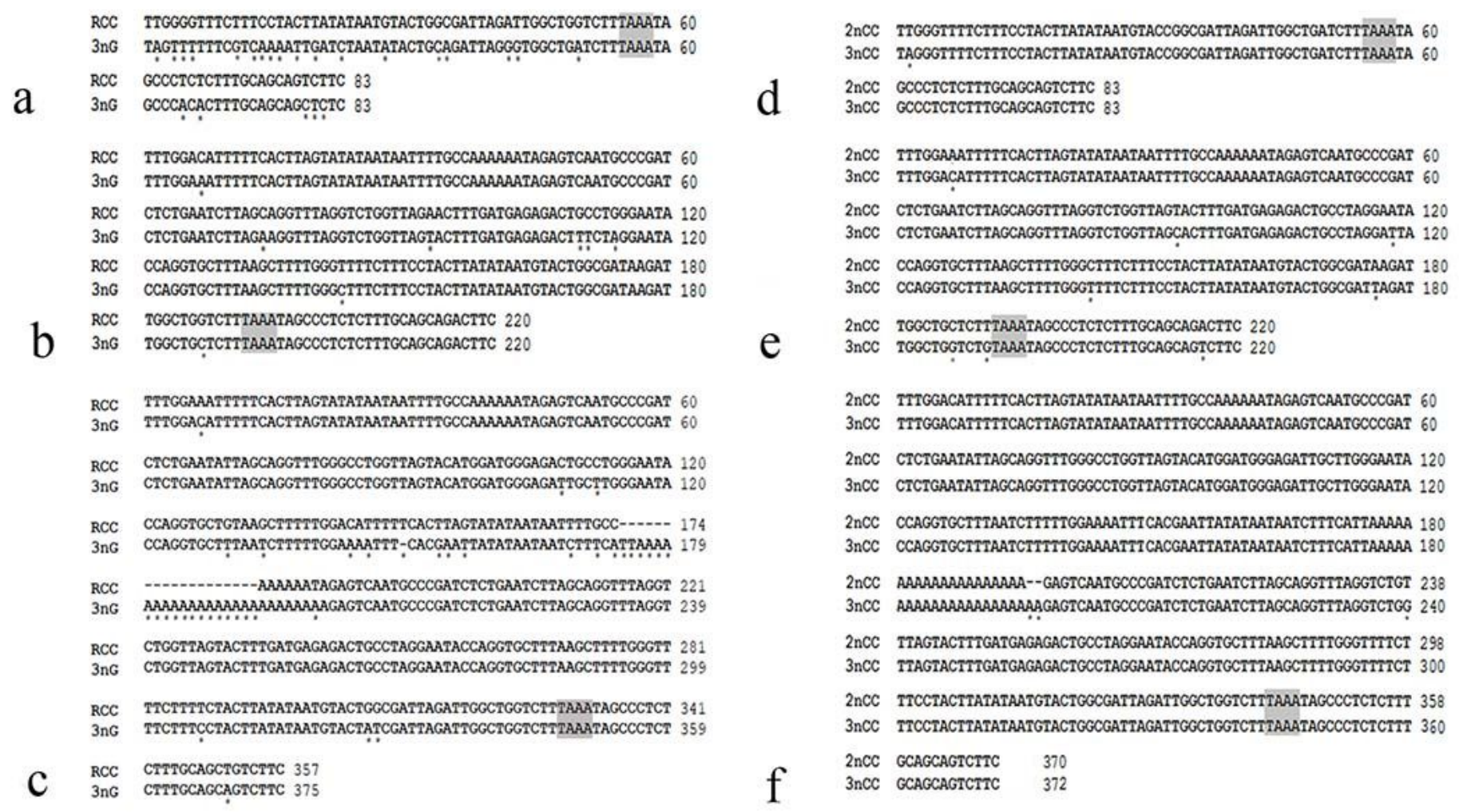

\section{Figure 5}

Comparison of the NTS sequences from RCC, $3 n G, 2 n C C$ and $3 n C C$ (a) NTS-I sequences from RCC and 3nG; (b) NTS-II from RCC and 3nG; (c) NTS-III from RCC and 3nG; (d) NTS-IV sequences from 2nCC and 3nCC; (e) NTS-V from 2nCC and 3nCC; (f) NTS-VI from 2nCC and 3nCC. The NTS upstream TATA elements are shaded; asterisks mark variable sites in the NTS. 


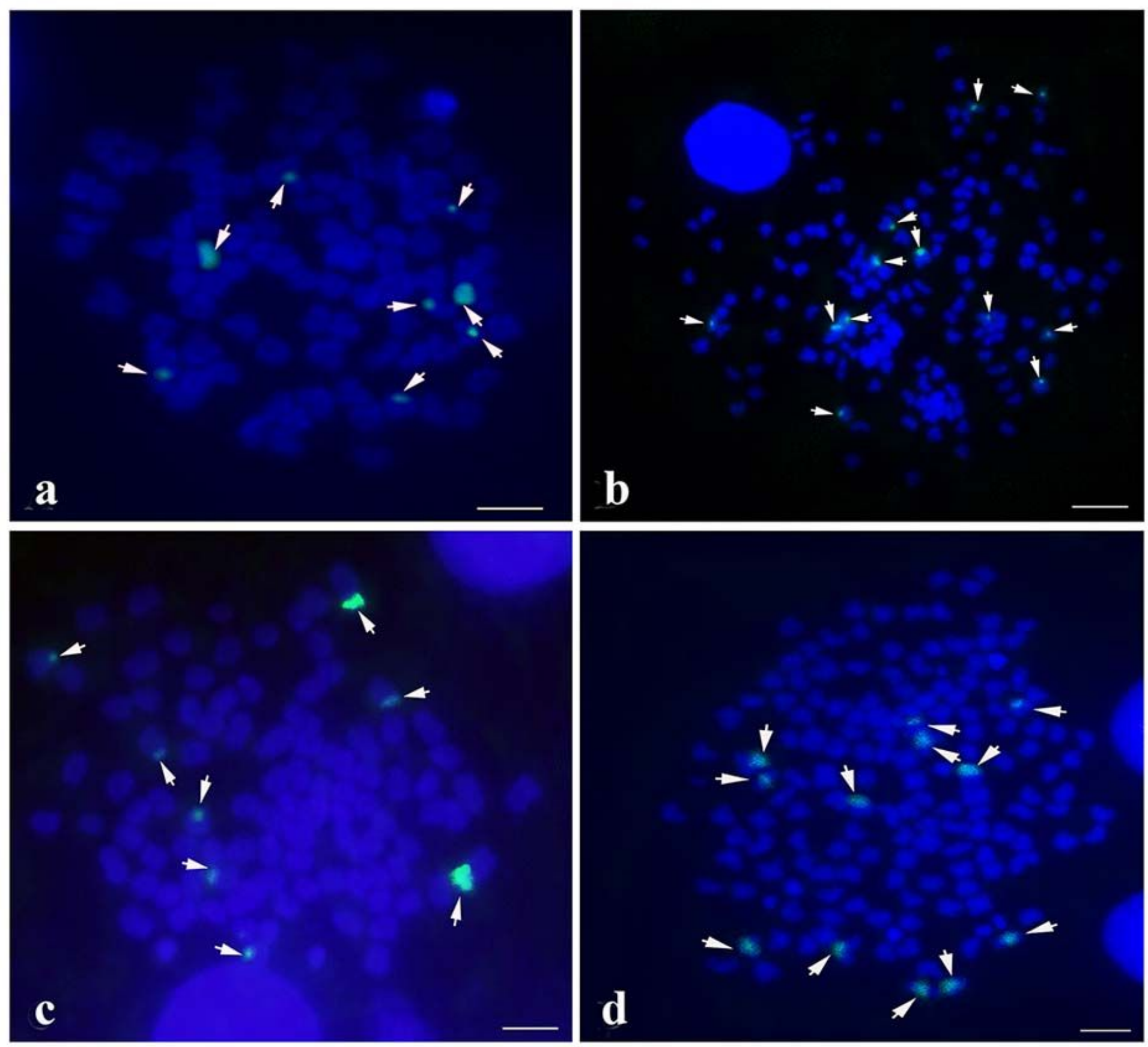

Figure 6

Examination of hybridizing signals by FISH (probes with $203 \mathrm{bp}$ ) in RCC, 3nG, 2nCC and 3nCC (a) There were eight $5 \mathrm{~S}$ gene loci (white arrows) in RCC; (b) There were twelve 5S gene loci (white arrows) in 3nG; (c) There were eight $5 \mathrm{~S}$ gene loci (white arrows) in 2nCC; (d) There were twelve $5 \mathrm{~S}$ gene loci (white arrows) in 3nCC. Bar $=3 \mu \mathrm{m}$. 


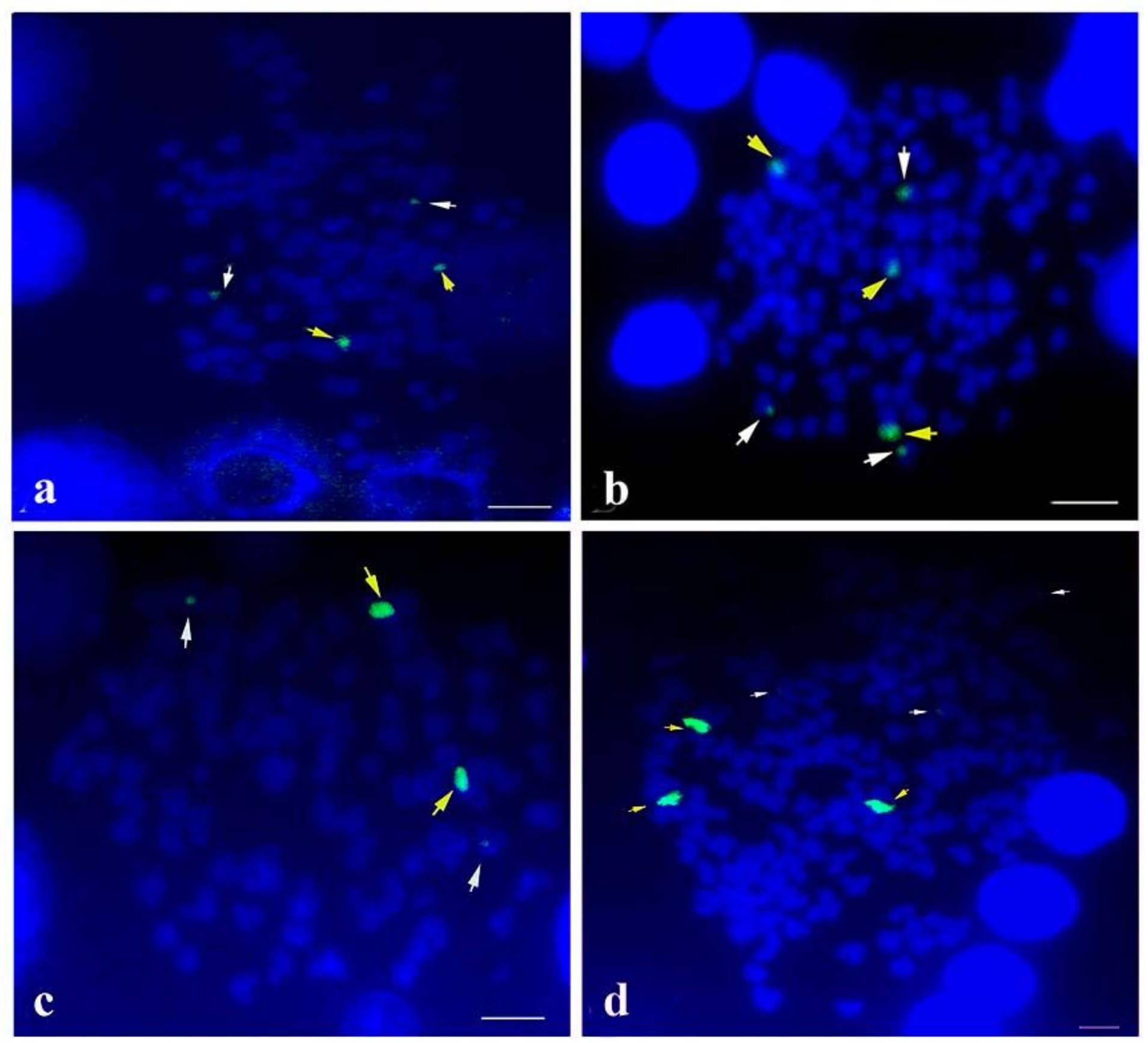

\section{Figure 7}

Examination of hybridizing signals by FISH (probes with 340 bp) in RCC, 3nG, 2nCC and 3nCC (a) There were two big (yellow arrows) and two small 5S gene loci (white arrows) in RCC; (b) There were three big (yellow arrows) and three small 5S gene loci (white arrows) in 3nG; (c) There were two big (yellow arrows) and two small $5 S$ gene loci (white arrows) in 2nCC; (d) There were three big (yellow arrows) and three small 5 S gene loci (white arrows) in 3nCC. Bar $=3 \mu \mathrm{m}$. 


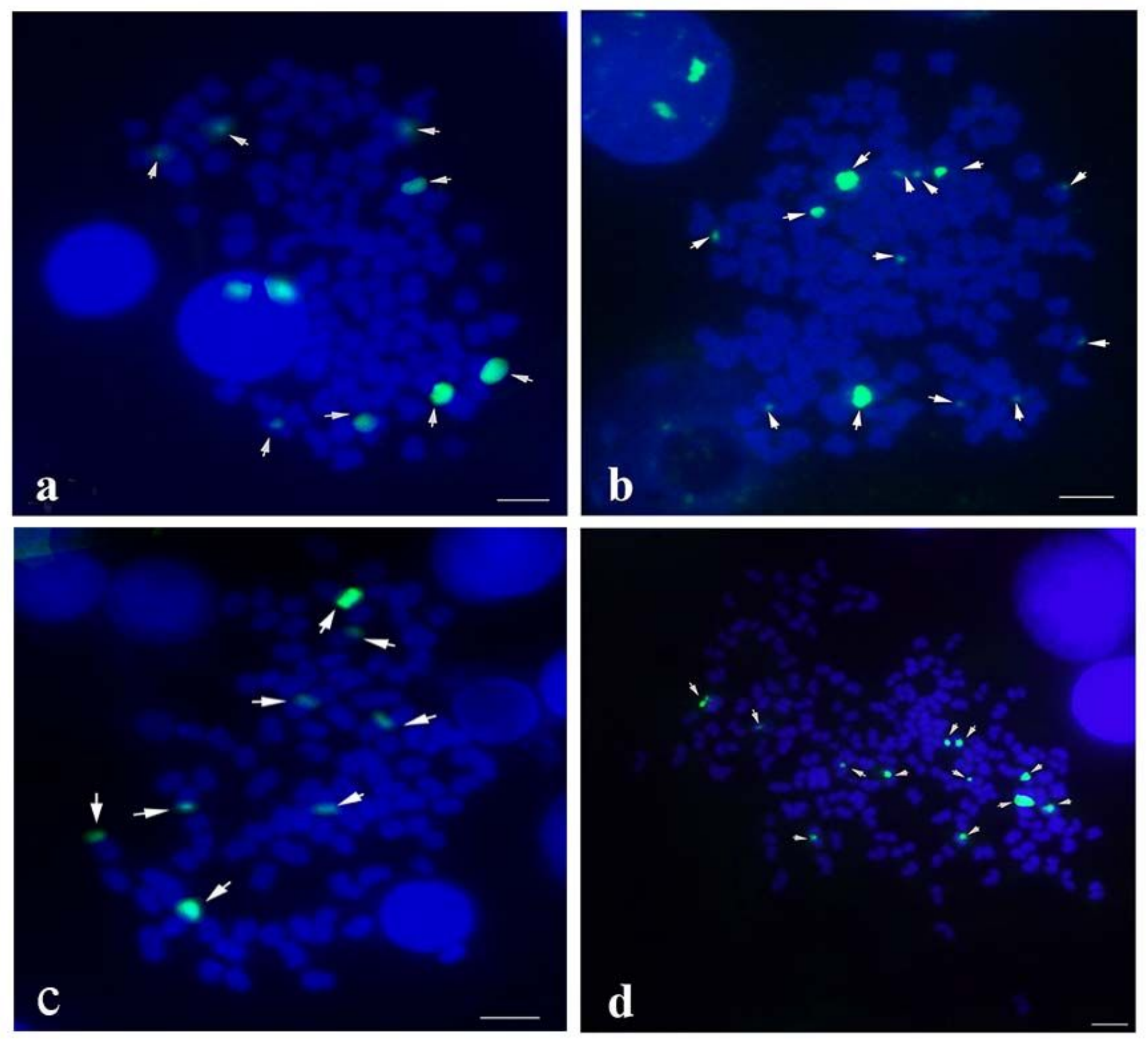

\section{Figure 8}

Examination of hybridizing signals by FISH in RCC and 3nG with 477 bp probes, in 2nCC and 3nCC with 490 bp probes (a) There were eight $5 S$ gene loci (white arrows) in RCC; (b) There were twelve 5 S gene loci (white arrows) in 3nG; (c) There were eight $5 \mathrm{~S}$ gene loci (white arrows) in 2nCC; (d) There were twelve $5 \mathrm{~S}$ gene loci (white arrows) in 3nCC. Bar $=3 \mu \mathrm{m}$. 